\title{
Relationship between body size of adult Anopheles gambiae s.l. and infection with the malaria parasite Plasmodium falciparum
}

\author{
E. O. LYIMO ${ }^{1}$ and J. C. KOELLA ${ }^{2 *}$ \\ ${ }^{1}$ Ifakara Centre, Box 53, Ifakara, Tanzania \\ ${ }^{2}$ Swiss Tropical Institute, Socinstrasse 57, CH-4002 Basel, Switzerland
}

(Received 2 Fuly 1991; revised 22 August 1991 ; accepted 23 August 1991)

SUMMARY

The influence of adult female body size of Anopheles gambiae s.l. on development of midgut and salivary gland infections by the parasite Plasmodium falciparum was investigated in a field study carried out in Tanzania. The proportion of mosquitoes infected during a blood meal was independent of size. However, the number of oocysts harboured by infected mosquitoes increased with size of the mosquito. The proportion of mosquitoes with sporozoites, and thus potentially infective to humans, was highest in intermediate-sized mosquitoes, whereas the largest and smallest mosquitoes were less likely to have sporozoites. This pattern is interpreted as a combination of high survival rate of large, uninfected mosquitoes and of low survival rate of mosquitoes infected with many oocysts.

Key words: Anopheles gambiae, body size, malaria, Plasmodium falciparum, oocyst, sporozoite, transmission.

\section{INTRODUCTION}

Variability within mosquito populations of factors affecting malaria transmission has received only little attention, although theoretical studies have shown that it affects the pattern of transmission (Dye \& Hasibeder, 1986; Kingsolver, 1987; Koella, 1991). One of the factors contributing to variability in transmission may be body size of the mosquito vector. Considerable variation in body size within populations has been observed for many mosquito species (Fish, 1985), including Anopheles gambiae (Gillies \& De Meillon, 1968), the main vector of malaria in Africa. In addition, body size has been found to affect transmission of several viral diseases. Small individuals of Culex tritaeniorhynchus are more likely to be infected with Japanese encephalitis virus (Takahashi, 1976) and West Nile virus (Baqar et al. 1980) during a blood meal than larger individuals. Grimstad \& Haramis (1984) have shown that nutritionally deprived larvae of Aedes triseriatus developed into small adults that are infected with $\mathrm{La}$ Crosse virus at higher rates than larger, wellnourished individuals.

The effect of body size on malaria transmission has only rarely been studied. Kitthawee, Ehman \& Sattabongkot (1990) artificially fed four size-classes of laboratory-reared Anopheles dirus with Plasmodium falciparum and showed that the largest size-

\footnotetext{
* Present address and address for reprint requests: Department of Biology, Imperial College, Prince Consort Road, London SW7 2BB, UK.
}

class developed the highest number of oocysts, though the proportion of infected mosquitoes was independent of size. In contrast, two earlier studies found no relationship between weight of Aedes aegypti and probability of infection by Plasmodium gallinaceum (Hovanitz, 1947) or between wing length of Anopheles stephensi and the number of oocysts of Plasmodium yoelii nigeriensis (Ichimori, 1989).

In the present study, we examine the relationship between adult size of the vector $A n$. gambiae and infection with $P$. falciparum. We examine oocysts as a measure of infection from humans to mosquitoes as well as sporozoites as a measure of infection from mosquitoes to humans.

\section{MATERIALS AND METHODS}

The study was carried out in Michenga, Kilombero District, southeastern Tanzania. The area is holo- to hyperendemic for malaria with peak transmission in June and July. The main malaria species is $P$. falciparum, occurring in about $95 \%$ of all infections, and its main vectors are $A n$. gambiae and $A n$. funestus. The study area has been described in detail by Tanner et al. (1987) and Biro (1987).

An. gambiae s.l. were sampled from 5 houses in July and the beginning of August, 1990. Female mosquitoes were caught as they were resting on the walls inside houses in the early morning. We assumed that blood-fed mosquitoes had fed only on humans because no animals were kept in the houses.

For oocyst detection freshly fed female mosquitoes were selected. They were fed on a $10 \%$ glucose 

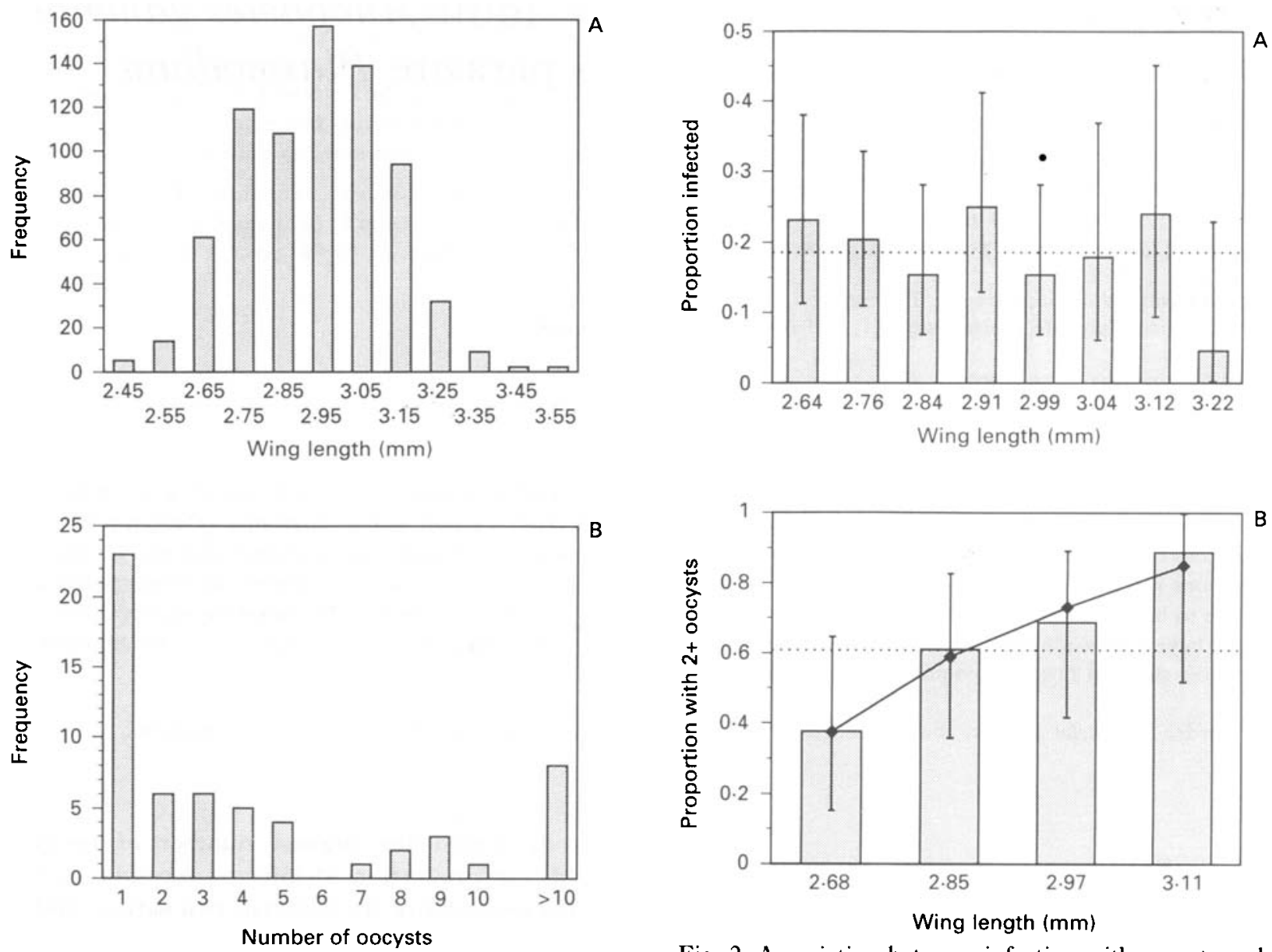

Fig. 1. Frequency distributions of (A) wing lengths and (B) oocyst numbers in infected mosquitoes. In (A) the wing lengths are grouped into $0.1 \mathrm{~mm}$ intervals and each interval is labelled with its midpoint.

solution for 5 days, when their abdomens were dissected and examined for oocysts. For sporozoite detection mosquitoes were killed immediately after capture. Their heads and thoraxes were investigated with an ELISA specific for the (NANP) $)_{40}$ repeat region of the circumsporozoite protein (Campbell et al. 1987). The cut-off value for the optical density separating infected from uninfected mosquitoes was set to the optical density of a control well on the same plate containing 500 sporozoites, which allows a compromise between the number of false positives and false negatives (N. Weiss, unpublished data).

The size of a mosquito was measured as the length of its wing, which correlated with dry weight (E. Lyimo, unpublished data). Wings were measured to the nearest $0.01 \mathrm{~mm}$ with an ocular micrometer from the distal end of the alula to the tip, excluding the fringe scales.

For the analysis of the proportions of mosquitoes with oocysts and with sporozoites the wing lengths were grouped into 8 size classes. The cut-off values between classes were determined so that the number of mosquitoes in each class was similar. A logistic regression, with wing length and the square of the

Fig. 2. Association between infection with oocysts and wing length. The $x$ axis is labelled with the mean wing length of the mosquitoes in each size-class. (A)

Proportions of mosquitoes infected in 8 size-classes. The vertical lines denote the $95 \%$ confidence intervals. The horizontal, dotted line denotes the overall proportion of infected mosquitoes. (B) Proportions of the infected mosquitoes with 2 or more oocysts in 4 size-classes. The measured proportions are shown as bars, the $95 \%$ confidence intervals as vertical lines. The horizontal, dotted line denotes the overall proportion of infected mosquitoes with 2 or more oocysts. The proportions predicted by a logistic regression (see text and Table 1) are shown as connected diamonds.

wing length as independent variables, tested for an association between wing length and proportions positive. The relationship between wing length and proportion infected, $f$, was thus estimated as the parameters of the model

$\ln \left(\frac{f}{1-f}\right)=A+B$ (wing length $)+C(\text { wing length })^{2}$.

Because the distribution of oocysts was overdispersed, with many mosquitoes harbouring only 1 oocyst, the relationship between number of oocysts and wing length could not be found with a product-moment correlation analysis. Therefore the relationship between wing length and the proportion of those infected mosquitoes harbouring 2 or more 
Table 1. Analysis of the relationship between wing length and proportions of mosquitoes infected $(a)$ with oocysts, $(b)$ with 2 or more oocysts, or $(c)$ with sporozoites

(The estimates are the parameters of a logistic regression. Thus the proportion of infected mosquitoes, $f$, was modelled as $\ln (f / 1-f)=A+B$ (wing length) $+C$ (wing length) ${ }^{2}$ for (a) and (c), where 8 classes were available, and as $\ln (f / 1-f)=A+B$ (wing length) for $(b)$, where only 4 size-classes were available.)

\begin{tabular}{|c|c|c|c|c|c|}
\hline & Source & D.F. & $x^{2}$ & $p$ & Estimate \\
\hline \multirow[t]{4}{*}{ (a) Oocyst } & Intercept & 1 & $0 \cdot 30$ & $0 \cdot 584$ & $23 \cdot 23$ \\
\hline & Wing length & 1 & $0 \cdot 30$ & 0.583 & $-16 \cdot 12$ \\
\hline & ${\text { (Wing length })^{2}}^{2}$ & 1 & $0 \cdot 34$ & 0.558 & $2 \cdot 965$ \\
\hline & Likelihood ratio & 5 & 4.88 & $0 \cdot 431$ & \\
\hline \multirow{3}{*}{ (b) $>1$ Oocyst } & Intercept & 1 & 5.99 & $0 \cdot 014$ & $14 \cdot 34$ \\
\hline & Wing length & 1 & $6 \cdot 34$ & $0 \cdot 012$ & $-5 \cdot 166$ \\
\hline & Likelihood ratio & 2 & $0 \cdot 32$ & 0.853 & \\
\hline \multirow[t]{4}{*}{ (c) Sporozoite } & Intercept & 1 & $11 \cdot 25$ & $<0.001$ & $109 \cdot 0$ \\
\hline & Wing length & 1 & $11 \cdot 55$ & $<0.001$ & $-76 \cdot 12$ \\
\hline & (Wing length) $^{2}$ & 1 & $12 \cdot 00$ & $<0.001$ & $13 \cdot 36$ \\
\hline & Likelihood ratio & 5 & $3 \cdot 47$ & 0.628 & \\
\hline
\end{tabular}

oocysts was analysed. Only infected mosquitoes were used for this analysis. So that size-classes had a sufficient number of mosquitoes, we formed 4 new size-classes by merging adjacent classes mentioned above. The relationship was found with a logistic regression with wing length as the independent variable. No quadratic term was included because only 4 size classes were involved. The logistic regressions were performed with the procedure CATMOD of the SAS package (SAS, 1988).

Because of the procedures described above, oocyst infection was measured as an incidence, i.e. as the proportion of mosquitoes that acquired a new infection during a blood meal, whereas sporozoite infection was measured as a prevalence, i.e. the proportion of mosquitoes that harboured sporozoites at a given time. Thus the two proportions are not directly comparable.

\section{RESULTS}

A total of 324 mosquitoes was dissected for oocysts and 425 investigated for sporozoites.

The wing lengths varied from 2.41 to $3.52 \mathrm{~mm}$ (Fig. 1 A) with a mean of $2.92 \mathrm{~mm}$ and a standard deviation of $0.175 \mathrm{~mm}$. It was found that $18.2 \%$ of the mosquitoes were infected with oocysts and in the infected mosquitoes the number of oocysts ranged from 1 to 49 (Fig. 1 B) with a mean of 5.3 and a median of $2 \cdot 5$. Of the infected mosquitoes $38.9 \%$ had only 1 oocyst. The proportion of mosquitoes with sporozoites was $28 \cdot 1 \%$.

The proportion of mosquitoes infected with oocysts (Fig. 2A) was independent of size (Table $1 a)$. In contrast, the proportion of mosquitoes infected with 2 or more oocysts (Fig. $2 \mathrm{~B}$ ) increased with wing length (Table $1 \mathrm{~b}$ ). In the smallest sizeclass $37.5 \%$ of the mosquitoes had more than

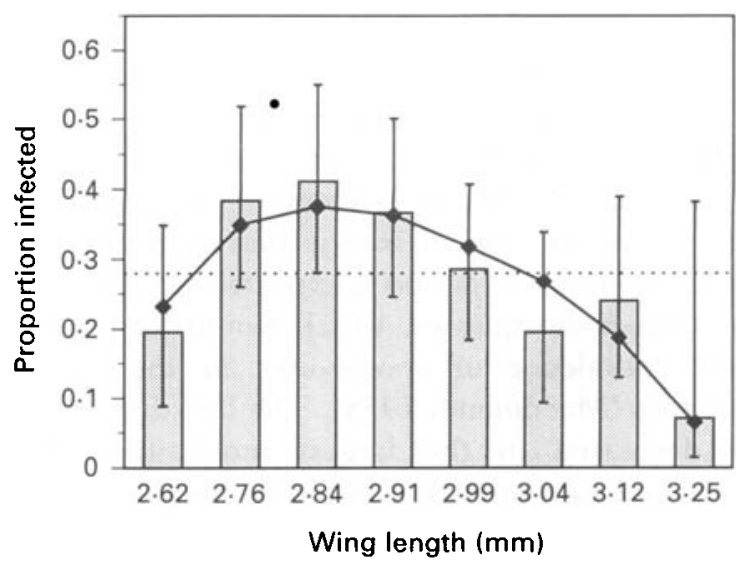

Fig. 3. Proportion of mosquitoes infected with sporozoites in 8 size-classes. The $x$ axis is labelled with the mean wing length of the mosquitoes in each size class category. The vertical lines denote the $95 \%$ confidence intervals. The horizontal, dotted line denotes the overall proportion of infected mosquitoes. The proportions predicted by a logistic regression (see text and Table 1) are shown as connected diamonds.

1 oocyst, whereas $88.9 \%$ of the mosquitoes in the largest size-class had more than 1 .

The proportion of mosquitoes with sporozoites increased from $19.5 \%$ in the smallest mosquitoes to $41.1 \%$ in intermediate size-classes and dropped again to $7.1 \%$ in the largest mosquitoes (Fig. 3 ). The non-linear relationship of wing length with proportion infected could be closely approximated with a quadratic function (Table $1 c$ ). The parameters of the logistic regression (Table $1 c$ ) estimated the highest proportion at $-B / 2 C=2.85 \mathrm{~mm}$, which was slightly below the mean wing length (Fig. 1 A). 


\section{DISCUSSION}

Adult size of An. gambiae affected key factors of transmission of the malaria parasite $P$. falciparum. Although the risk of becoming infected during a bloodmeal was independent of size, mosquitoes tended to develop many oocysts only if they were large. This pattern was similar to that observed in laboratory-reared mosquitoes (Kitthawee et al. 1990) and might have been due to differences in size of the bloodmeal. Size of the bloodmeal is positively correlated with body size in several mosquito species (Reisen, 1975; Ichimori, 1989; Kitthawee et al. 1990), including An. gambiae (P. Billingsley, unpublished data). For the low gametocyte densities encountered in human infections this leads to a positive correlation between size of the mosquito, number of ingested gametocytes, and number of oocysts (Carter \& Graves, 1988; T. Ponnudurai, personal communication).

Intermediate-sized mosquitoes were most likely to harbour sporozoites. The peak sporozoite infection was observed for mosquitoes close to the mean size in the population. This result is contrary to the expectation. Because adult survival generally increases with body size (Nasci, 1986; Packer \& Corbet, 1989; Landry, Defoliart \& Hogg, 1988; Haramis, 1985 ), the proportion of mosquitoes surviving the parasite's incubation period is expected to be highest in the largest mosquitoes, which would lead to the highest prevalence of sporozoites in the largest mosquitoes (Macdonald, 1958). The fact that prevalence decreased in the largest mosquitoes thus implies increased mortality in large mosquitoes. We could speculate that this increased mortality was due to the large number of oocysts found in the large mosquitoes. Although investigations of the pathogenicity of malaria parasites in mosquitoes are not conclusive, recent studies (Gad, Maier \& Piekarski, 1979; Klein et al. 1982) have shown that survival is reduced in infected mosquitoes, in particular in those mosquitoes harbouring more than 10 oocysts (Klein et al. 1986).

Whatever the processes leading to the observed patterns, mosquito size has a strong effect on the number of gametocytes that develop into oocysts and sporozoites. Thus, mosquito size might influence the rate at which malaria parasites are transmitted.

We thank Derek Charlwood, Jo Lines, Mike Packer and Andy Read for helpful discussions and Drs Chris Dye, Willem Takken, Peter Billingsley for a critical review of an earlier version of the manuscript. The entomology team at STIFL helped with mosquito collections. E. Lyimo was supported by the Directorate General for International Cooperation of the Government of the Netherlands. The study was undertaken as part of the Kilombero Malaria Project, a joint project of the World Health Organization and of the Governments of Tanzania, Switzerland, and the Netherlands.

\section{REFERENCES}

BAQAR, W., HAYES, T. \& AHMED, T. (1980). The effect of larval rearing conditions and adult age on the susceptibility of Culex tritaeniorhynchus to infection with West Nile virus. Mosquito News 40, 165-71. BIRO, s. (1987). Investigations on the bionomics of Anopheline vectors in the Ifakara area (Kilombero District, Tanzania). Ph.D. thesis, University of Basel. CAMPBELL, G. H., BRANDLING-BENNETT, A. D., ROBERTS, J. M., COlliNs, F. H., KASEJE, D. C. O., BARBER, A. M. \& TURNER, A. (1987). Detection of antibodies in human sera to the repeating epitope of the circumsporozoite protein of Plasmodium falciparum using the synthetic peptide (NANP) $)_{3}$ in an enzyme-linked immunosorbent assay (ELISA). American Fournal of Tropical Medicine and Hygiene 37, 17-21.

CARTER, R. \& GRAves, P. M. (1988). Gametocytes. In Malaria: Principles and Practice of Malariology (ed. Wernsdorfer, W. H. \& McGregor, I.), pp. 253-305. Edinburgh: Churchill Livingstone.

DYE, C. \& HASIBEDER, G. (1986). Population dynamics of mosquito-borne disease: effects of flies which bite some people more frequently than others. Transactions of the Royal Society of Tropical Medicine and Hygiene $80,69-77$.

FISH, D. (1985). An analysis of adult size variation within natural mosquito populations. In Ecology of Mosquitoes: Proceedings of a Workshop (ed. Lounibos, L. P., Rey, J. R. \& Frank, J. H.), pp. 419-429. Florida Medical Entomological Laboratory. GAD, A. M., MAIER, W. A. \& PIEKARSKI, G. (1979). Pathology of Anopheles stephensi after infection with Plasmodium berghei berghei. I. Mortality rate. Zeitschrift für Parasitenkunde 60, 249-61.

gillies, M. T. \& DE Meillon, B. (1968). The Anophelinae of Africa South of the Sahara, 2nd edn. Publication no. 54. Johannesburg: South African Institute for Medical Research.

GRIMSTAD, P. R. \& HARAMIS, L. D. (1984). Aedes triseriatus (Diptera: Culicidae) and La Crosse virus. III. Enhanced oral transmission by nutrition-deprived mosquitoes. Fournal of Medical Entomology 21, 229-62.

HARAMIS, L. D. (1985). Larval nutrition, adult body size, and the biology of Aedes triseriatus. In Ecology of Mosquitoes: Proceedings of a Workshop (ed. Lounibos, L. P., Rey, J. R. \& Frank, J. H.), pp. 431-437. Florida Medical Entomological Laboratory. Hovanitz, w. (1947). Physiological factors which influence the infection of Aedes aegypti with Plasmodium gallinaceum. American Fournal of Hygiene 45, 67-81.

ICHIMORI, K. (1989). Correlation of mosquito size, blood meal size and malarial oocyst production. Fapanese Fournal of Sanit Zoology 2, 81-5.

KINGsolver, J. G. (1987). Mosquito host choice and the epidemiology of malaria. American Naturalist 130 , 811-27.

KitThaWeE, S., EHMAN, J. D. \& SATtabongkot, J. (1990). Evaluation of survival potential and malaria susceptibility among different size classes of laboratory-reared Anopheles dirus. American Fournal of Tropical Medicine and Hygiene 43, 328-32. 
KLEIN, T. A., HARRISON, B. A., ANDRE, R. G., WHITMIRE, R. E. \& INLAO, I. (1982). Detrimental effects of Plasmodium cynomolgi infections on the longevity of Anopheles dirus. Mosquito News 42, 265-71.

KLEIN, T. A., HARRISON, B. A., GROVE, J. S., DIXON, S. V. \& ANDRE, R. G. (1986). Correlation of survival; rates of Anopheles dirus A (Diptera: Culicidae) with different infection densities of Plasmodium cynomolgi. Bulletin of the World Health Organization 64, 901-7.

KOELLA, J. C. (1991). On the use of mathematical models of malaria transmission. Acta Tropica 49, 1-25.

LANDRY, s. v., DEFOLIART, G. R. \& HOGG, D. B. (1988).

Adult body size and survivorship in a field population of Aedes triseriatus. Fournal of the American Mosquito Control Association 4, 121-8.

Macdonald, G. (1957). The Epidemiology and Control of Malaria. London: Oxford University Press.

NASCI, R. s. (1986). The size of emerging and hostseeking Aedes aegypti and the relation of size to blood- feeding success in the field. Fournal of the American Mosquito Control Association 2, 61-2.

PACKer, M. J. \& CORBET, P. S. (1989). Size variation and reproductive success of female Aedes punctor (Diptera: Culicidae). Ecological Entomology 14, 297-309. REISEN, W. K. (1975). Intraspecific competition in Anopheles stephensi Liston. Mosquito News 35, 473-82. SAS. (1988). SAS/STAT User's Guide, Release 6.03 Edition. SAS Institute Inc., Cary, NC, USA.

TAKAHASHI, M. (1976). The effects of environmental and physiological conditions of Culex tritaeniorhynchus on the pattern of transmission of Japanese encephalitis virus. Fournal of Medical Entomology 13, 275-84.

TANNER, M., DEGREMONT, A., DE SAVIGNY, D., FREYVogel, T., MaYombana, c. \& TAYARI, s. (1987). Longitudinal study on the health status of children in Kikwawila village, Tanzania: study area and design. Acta Tropica 44, 137-74. 